\title{
The Impact of Serum Glucose on the Predictive Value of Serum Lactate for Hospital Mortality in Critically Ill Surgical Patients
}

\author{
Xue Chen $\mathbb{D}^{1}{ }^{1}$ Jianbin Bi $\mathbb{D}^{1,2}$ Jia Zhang $\mathbb{D}^{1,2}$ Zhaoqing Du $\mathbb{D}^{1,2}$ Yifan Ren ${ }^{1,}{ }^{1,2}$ \\ Shasha Wei $\mathbb{D}^{1},{ }^{1}$ Fenggang Ren, ${ }^{1,2}$ Zheng Wu $\mathbb{D}^{2}{ }^{2}$ Yi Lv $\mathbb{D}^{1,2}$ and Rongqian $W u \mathbb{D}^{1}$ \\ ${ }^{1}$ National Local Joint Engineering Research Center for Precision Surgery \& Regenerative Medicine, Shaanxi Provincial Center for \\ Regenerative Medicine and Surgical Engineering, First Affiliated Hospital of Xi'an Jiaotong University, Xi'an, \\ Shaanxi Province, China \\ ${ }^{2}$ Department of Hepatobiliary Surgery, First Affiliated Hospital of Xi'an Jiaotong University, Xi'an, Shaanxi Province, China
}

Correspondence should be addressed to Rongqian Wu; rwu001@mail.xjtu.edu.cn

Received 2 April 2019; Accepted 25 September 2019; Published 26 November 2019

Academic Editor: Carlo Chiarla

Copyright (c) 2019 Xue Chen et al. This is an open access article distributed under the Creative Commons Attribution License, which permits unrestricted use, distribution, and reproduction in any medium, provided the original work is properly cited.

Background. Lactate has been widely used as a risk indicator of outcomes in critically ill patients due to its ready measurement and good predictive ability. However, the interconnections between lactate metabolism and glucose metabolism have not been sufficiently explored, yet. In this study, we aimed to investigate whether glucose levels could influence the predictive ability of lactate and design a more comprehensive strategy to assess the in-hospital mortality of critically ill patients. Methods. We analyzed the clinical data of 293 critically ill patients. The primary outcome was in-hospital mortality. The logistic regression analysis and the area under the receiver operating characteristic curve (AUROC) were applied to evaluate the predictive ability of lactate in association with glucose. Results. The lactate level showed significant association with in-hospital mortality, and its predictive ability was also comparable to other prognostic scores such as the SOFA score and APACHE II score. We further divided 293 patients into three groups based on glucose levels: low-glucose group $(<7 \mathrm{mmol} / \mathrm{L})$, medium-glucose group (7$9 \mathrm{mmol} / \mathrm{L})$, and high-glucose group $(>9 \mathrm{mmol} / \mathrm{L})$. The lactate level was associated with in-hospital mortality in the low- and high- glucose groups, but not in the medium-glucose group, whereas the SOFA score and APACHE II score were associated with in-hospital mortality in all three glucose groups. The AUROC of lactate in the medium-glucose group was also the lowest among the three glucose groups, indicating a decrease in its predictive ability. Conclusions. Our findings demonstrated that the predictive ability of lactate to assess in-hospital mortality could be influenced by glucose levels. In the medium glucose level (i.e., $7-9 \mathrm{mmol} / \mathrm{L}$ ), lactate was inadequate to predict in-hospital mortality and the SOFA score; the APACHE II score should be utilized as a complementation in order to obtain a more accurate prediction.

\section{Background}

An elevated lactate level is a well-known predictor of organ dysfunction and mortality in critically ill patients [1-10]. The pathways for glucose and lactate metabolism are deeply interconnected. In the Cori cycle, glucose can be converted to lactate though glycolysis and lactate can generate glucose through gluconeogenesis, indicating that glucose can greatly influence lactate metabolism and vice versa. In this regard, blood glucose levels may be a confounder in the association between high lactate levels and increased mortality in ICU patients. Some studies have demonstrated the associations of glucose level and lactate level with the risk of death $[1,3$, 5, 7-17]. A recent study has shown that a low glucose level combined with a high lactate level was associated with the highest mortality of ICU patients [11]. However, whether glucose levels influence the predictive value of lactate for hospital mortality in critically ill patients remained largely unknown. Herein, we conducted a study to investigate the predictive ability of lactate at various glucose levels. Specifically, we hypothesized that glucose levels could modify the association between high lactate levels and increased mortality in critically ill patients. We aimed to get a more comprehensive appreciation and a more accurate prediction strategy 
TABLE 1: Patient characteristics, reason for ICU admission and comorbidities.

\begin{tabular}{|c|c|c|c|c|}
\hline Variable & $\begin{array}{l}\text { All patients } \\
(n=293)\end{array}$ & $\begin{array}{l}\text { Hospital survivors } \\
\qquad(n=234)\end{array}$ & $\begin{array}{l}\text { Hospital nonsurvivors } \\
\qquad(n=59)\end{array}$ & $p$ value \\
\hline Age & 61.44 & 60.87 & 63.71 & 0.306 \\
\hline Male & $62.8 \%(184)$ & $62.7 \%(147)$ & $62.8 \%(37)$ & 0.988 \\
\hline \multicolumn{5}{|l|}{ Reason for ICU admission } \\
\hline Cardiovascular disease & $17.4 \%(51)$ & $15.4 \%(36)$ & $25.4 \%(15)$ & 0.069 \\
\hline Gastrointestinal disease & $27.6 \%(81)$ & $25.6 \%(60)$ & $35.6 \%(21)$ & 0.127 \\
\hline Neurological disease & $14.7 \%(43)$ & $13.7 \%(32)$ & $18.6 \%(11)$ & 0.335 \\
\hline Respiratory disease & $26.3 \%(77)$ & $25.6 \%(60)$ & $28.8 \%(17)$ & 0.621 \\
\hline Surgery & $34.5 \%(101)$ & $34.6 \%(81)$ & $33.9 \%(20)$ & 0.918 \\
\hline Sepsis & $13.7 \%(40)$ & $14.1 \%(33)$ & $11.9 \%(7)$ & 0.655 \\
\hline Trauma & $10.6 \%(31)$ & $11.5 \%(27)$ & $6.8 \%(4)$ & 0.288 \\
\hline Other & $6.5 \%(19)$ & $5.1 \%(12)$ & $11.9 \%(7)$ & 0.060 \\
\hline \multicolumn{5}{|l|}{ Comorbidities } \\
\hline ACS & $10.6 \%(31)$ & $9.4 \%(22)$ & $15.3 \%(9)$ & 0.192 \\
\hline AKI & $16.7 \%(49)$ & $17.1 \%(40)$ & $15.3 \%(9)$ & 0.735 \\
\hline ALI & $10.6 \%(31)$ & $10.3 \%(24)$ & $11.9 \%(7)$ & 0.720 \\
\hline ARDS & $13.3 \%(39)$ & $12.4 \%(29)$ & $16.9 \%(10)$ & 0.357 \\
\hline Cirrhosis & $8.9 \%(26)$ & $9.4 \%(22)$ & $6.8 \%(4)$ & 0.572 \\
\hline Diabetes mellitus & $14.0 \%(41)$ & $11.5 \%(27)$ & $23.7 \%(14)$ & 0.016 \\
\hline DIC & $1.4 \%(4)$ & $1.3 \%(3)$ & $1.7 \%(1)$ & 0.807 \\
\hline Drinking & $22.5 \%(69)$ & $25.6 \%(60)$ & $10.2 \%(9)$ & 0.093 \\
\hline Hypertension & $28.3 \%(83)$ & $29.1 \%(68)$ & $25.4 \%(15)$ & 0.580 \\
\hline Malignant disease & $22.1 \%(65)$ & $21.8 \%(51)$ & $23.7 \%(14)$ & 0.749 \\
\hline MOF & $16.7 \%(49)$ & $14.1 \%(33)$ & $27.1 \%(16)$ & 0.017 \\
\hline Smoking & $32.4 \%(95)$ & $34.2 \%(80)$ & $25.4 \%(15)$ & 0.199 \\
\hline Stroke & $13.0 \%(38)$ & $12.8 \%(30)$ & $13.6 \%(8)$ & 0.880 \\
\hline APACHE II at ICU admission & 16.55 & 15.46 & 20.90 & $<0.001$ \\
\hline Glucose level at ICU admission & 8.02 & 8.06 & 7.85 & 0.505 \\
\hline Lactate level at ICU admission & 3.82 & 2.81 & 5.64 & $<0.001$ \\
\hline qSOFA score at ICU admission & 1.29 & 1.06 & 1.39 & 0.008 \\
\hline SOFA score at ICU admission & 8.02 & 7.33 & 10.76 & $<0.001$ \\
\hline Length of ICU stay & 14.62 & 13.91 & 17.42 & 0.355 \\
\hline
\end{tabular}

$p$ values generated by either Mann-Whitney or $\chi^{2}$ test. ACS: acute coronary syndrome; AKI: acute kidney injury; ALI: acute lung injury; ARDS: acute respiratory distress syndrome; DIC: disseminated intravascular coagulation; IHD: ischemic heart disease; APACHE II: Acute Physiology and Chronic Health Evaluation II; SOFA: Sequential Organ Failure Assessment; qSOFA: Quick Sequential Organ Failure Assessment.

of using lactate as a prognostic biomarker to assess the outcomes of ICU patients.

\section{Methods}

2.1. Patients and Data Sources. We conducted a retrospective study of 293 patients older than 18 years of age admitted to the intensive care unit (ICU) of The First Affiliated Hospital of Xi' an Jiaotong University from June 2013 to Dec 2016. The study was approved by the medical ethics committee of Xi' an Jiaotong University. The patient's informed written consent was waived due to the retrospective nature of this study. Anonymized patient information was obtained from the hospital's electronic patient database. The following data were recorded from the electronic medical record: demographics, comorbidities, initial and worst vital signs, and laboratory measurements. The APACHE II score, SOFA score, and qSOFA score were calculated according to References [1820]. Renal dysfunction was defined by the presence of acute kidney injury (AKI) according to the Kidney Disease Improving Global Outcomes (KDIGO) criteria [21].

2.2. Statistical Analysis. Continuous data was tested for normality by the Kolmogorov-Smirnov test. Normal distribution variables are reported as the means \pm standard deviations (SD) and compared by Student's $t$-test. Abnormal distribution variables are reported as the medians (interquartile range (IQR)) and compared by the Mann-Whitney ranksum test. Categorical variables are reported as the numbers and percentages and compared by the chi-squared analysis or Fisher's exact test. $p<0.05$ was considered to be statistically significant. The logistic regression analysis and the area 
TABLE 2: Univariate and multivariate analyses of in-hospital mortality.

\begin{tabular}{|c|c|c|c|c|}
\hline \multirow{2}{*}{ Variable } & \multicolumn{2}{|c|}{ Univariable regression } & \multicolumn{2}{|c|}{ Multivariable regression } \\
\hline & OR $(95 \%-C I)$ & $p$ value & OR $(95 \%-C I)$ & $p$ value \\
\hline Age & $1.010(0.993-1.027)$ & 0.266 & & \\
\hline Gender & $0.995(0.551-1.797)$ & 0.988 & & \\
\hline \multicolumn{5}{|l|}{ Reason for ICU admission } \\
\hline Cardiovascular disease & $1.875(0.945-3.720)$ & 0.072 & & \\
\hline Gastrointestinal disease & $0.624(0.240-1.147)$ & 0.129 & & \\
\hline Neurological disease & $1.447(0.681-3.074)$ & 0.337 & & \\
\hline Respiratory disease & $1.174(0.622-1.174)$ & 0.621 & & \\
\hline Surgery & $0.969(0.530-1.770)$ & 0.918 & & \\
\hline Sepsis & $0.820(0.343-1.959)$ & 0.655 & & \\
\hline Trauma & $0.558(0.187-1.661)$ & 0.294 & & \\
\hline Other & $2.490(0.935-6.634)$ & 0.068 & & \\
\hline \multicolumn{5}{|l|}{ Comorbidities } \\
\hline ACS & $1.735(0.753-3.996)$ & 0.196 & & \\
\hline AKI & $0.873(0.397-1.918)$ & 0.735 & & \\
\hline ALI & $1.178(0.481-2.883)$ & 0.720 & & \\
\hline ARDS & $1.443(0.659-3.158)$ & 0.359 & & \\
\hline Cirrhosis & $0.701(0.232-2.118)$ & 0.529 & & \\
\hline Diabetes mellitus & $2.385(1.159-4.908)$ & 0.018 & $2.326(1.055-5.130)$ & 0.036 \\
\hline DIC & $1.328(1.36-12.998)$ & 0.808 & & \\
\hline Drinking & $0.522(0.242-1.125)$ & 0.097 & & \\
\hline Hypertension & $0.832(0.434-1.595)$ & 0.580 & & \\
\hline Malignant disease & $1.116(0.568-2.193)$ & 0.749 & & \\
\hline MOF & $2.266(1.146-4.482)$ & 0.019 & $1.265(0.590-2.715)$ & 0.545 \\
\hline Smoking & $0.656(0.344-1.251)$ & 0.201 & & \\
\hline Stroke & $1.067(0.461-2.466)$ & 0.880 & & \\
\hline APACHE II & $1.093(1.052-1.135)$ & $<0.001$ & $1.065(1.007-1.128)$ & 0.027 \\
\hline Glucose & $0.990(0.928-1.056)$ & 0.795 & & \\
\hline Lactate & $1.131(1.065-1.200)$ & $<0.001$ & $1.093(1.024-1.168)$ & 0.008 \\
\hline qSOFA & $1.547(1.108-2.159)$ & 0.010 & $0.855(0.545-1.343)$ & 0.497 \\
\hline SOFA & $1.160(1.083-1.242)$ & $<0.001$ & $1.057(0.979-1.142)$ & 0.157 \\
\hline
\end{tabular}

under the receiver operating characteristic curve (AUROC) were applied to evaluate the predictive ability of lactate. All statistics analyses were performed using IBM SPSS version 23.0 for Windows (IBM, Chicago, Ill., United States).

\section{Results}

3.1. Patient Characteristics. We analyzed the data of 293 patients admitted to the ICU between June 2013 and Dec 2016. Of these patients, 59 (20.1\%) died during hospital stay. Patient characteristics of hospital survivors and nonsurvivors are shown in Table 1 . There were no statistically significant differences in age, gender, and reason for ICU admission between survivors and nonsurvivors. In terms of comorbidities, the deceased patients were more likely to have diabetes mellitus and multiple organ failure (MOF) than survivors. In addition, the lactate level, SOFA score, and APACHE II score at ICU admission were significantly higher in nonsurvivors than in survivors. However, there was no significant dif- ference in blood glucose levels between the hospital survivors and nonsurvivors.

3.2. Associations of Patient's Clinical Characteristics and Predictive Scores with In-Hospital Mortality. We firstly conducted univariable logistic regression analysis to investigate the associations of patients' clinical characteristics and predictive scores (including lactate level, glucose level, SOFA score, APACHE II score, and qSOFA score) with in-hospital mortality. As shown in Table 2, patients' age, gender, and reason for ICU admission were unassociated with in-hospital mortality. Among 15 various comorbidities, patients with diabetes mellitus and patients with MOF correlated significantly with higher in-hospital mortality. Among the five predictive scores, lactate level, SOFA score, APACHE II score, and qSOFA score were strongly associated with in-hospital mortality whereas the glucose level was not associated with in-hospital mortality. We further conducted a multivariate logistic regression analysis using the parameters shown to have statistical significance by univariate analysis. As a result, 


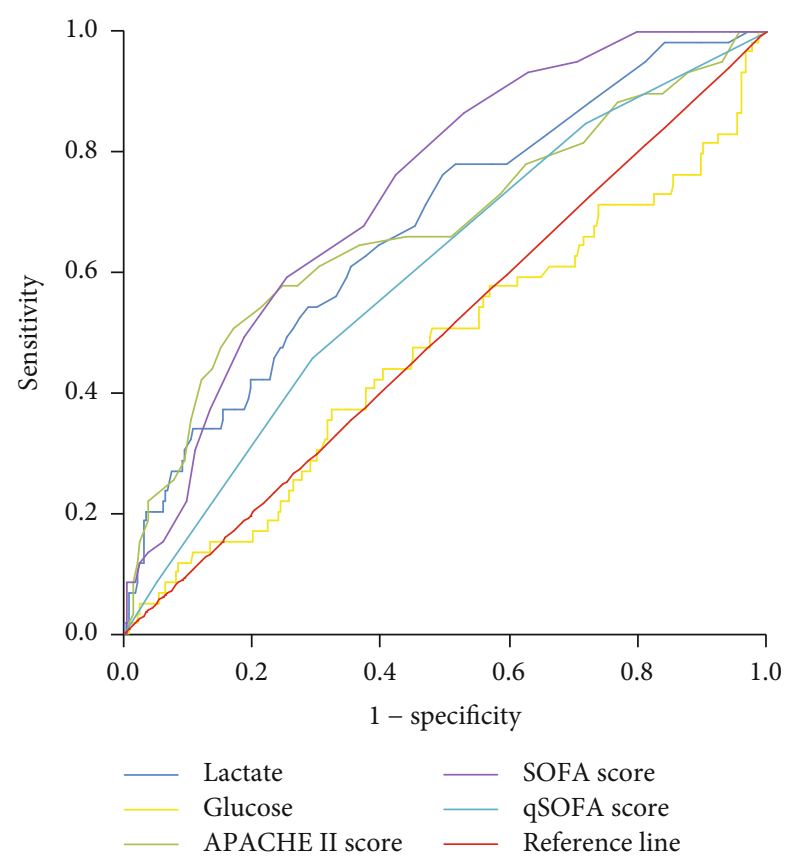

Figure 1: ROC of lactate, glucose, APACHE II score, SOFA score, and qSOFA score. The ROC of the five indexes were plotted, and their AUROC were compared. AUROC of lactate, glucose, APACHE II score, SOFA score, and qSOFA score were 0.678, $0.472,0.675,0.736$, and 0.605 , respectively.

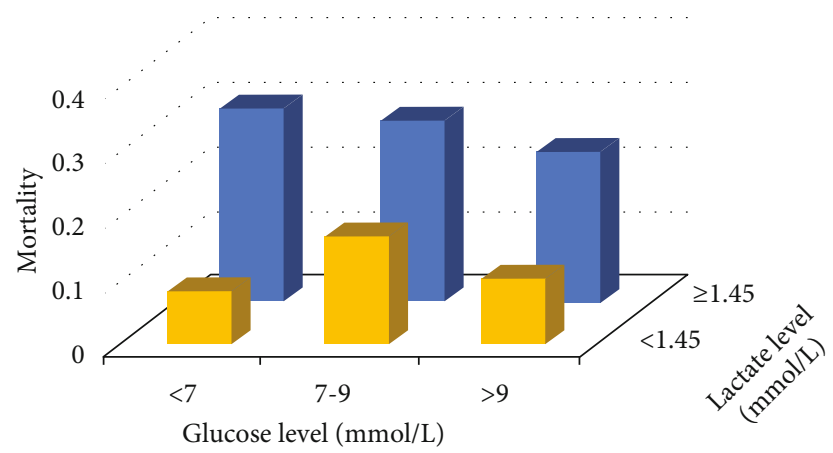

Figure 2: Combined effect of lactate and glucose on in-hospital mortality. The highest mortality was observed in the group with the lowest glucose level and the highest lactate level.

only diabetes mellitus, APACHE II, and lactate level were correlated significantly with higher in-hospital mortality.

The receiver operating characteristic (ROC) curve was also applied in our study to assess the mortality prediction performance of the five predictive scores. As seen in Figure 1, the AUROC of lactate level, glucose level, APACHE II score, SOFA score, and qSOFA score were $0.678,0.472$, $0.675,0.736$, and 0.605 , respectively. The AUROC of lactate was the second largest and comparable to that of the SOFA score. The calculated cutoff for lactate to predict in-hospital mortality was $1.45 \mathrm{mmol} / \mathrm{L}$. The AUROC of glucose, on the other hand, was much lower than that of the other four scores.

3.3. Glucose Levels Influence the Predictive Ability of Lactate. It has been reported that the glucose showed a U-shaped characteristic in a number of illnesses $[12,19]$. The "safe range" of glucose has been defined between $7.0 \mathrm{mmol} / \mathrm{L}$ and $9.0 \mathrm{mmol} / \mathrm{L}$ (the conversion factor of glucose is $1 \mathrm{mmol} / \mathrm{L}=$ $18 \mathrm{mg} / \mathrm{dL}$ ) in critically ill patients $[11,12]$. Following these criteria, we divided the 293 patients into three groups based on the glucose levels: low-glucose group (glucose $<7 \mathrm{mmol} / \mathrm{L}$ ), medium-glucose group (glucose: $7-9 \mathrm{mmol} / \mathrm{L}$ ), and highglucose group (glucose $>9 \mathrm{mmol} / \mathrm{L}$ ). Each group was further divided into two subgroups based on lactate cutoff point as shown in Table S1 (i.e., $1.45 \mathrm{mmol} / \mathrm{L}$; the conversion factor of lactate is $1 \mathrm{mmol} / \mathrm{L}=9 \mathrm{mg} / \mathrm{dL}$ ). The association of lactate levels with in-hospital mortality and the predictive ability of lactate were investigated in this scenario. As seen in Figure 2, the highest mortality was observed in the group with the lowest glucose level and the highest lactate level. The lactate level was associated with in-hospital mortality in the low- and high-glucose groups $(p<0.001)$, but not in the medium-glucose group (Table 3). The AUROC of lactate in the three glucose groups were $0.723,0.603$, and 0.677 , respectively. The AUROC of lactate in the medium-glucose group was also the lowest among the three (Figure 3).

\subsection{APACHE II Score and SOFA Score Could Be Utilized as} an Alternative Predictor of Hospital Mortality in the Medium-Glucose Group. In addition to lactate, the association between patients' clinical characteristics and other predictive scores (including APACHE II score, SOFA score, and qSOFA score) with in-hospital mortality in the three glucose groups was also investigated. As seen in Table 3 , only the SOFA score and APACHE II score were clearly associated with higher in-hospital mortality in all three glucose groups whereas the association of other indexes with mortality was influenced by glucose levels. The ROC and AUROC of the SOFA score, APACHE II score, and qSOFA score were also plotted and compared with that of lactate as show in Figure 4. In the medium-glucose group where the lactate level was unassociated with in-hospital mortality, the APACHE II score presented the largest AUROC, followed by the SOFA score, qSOFA, score and lactate.

\section{Discussion}

In this study, we demonstrated that glucose levels could influence the association between lactate and in-hospital mortality in critically ill patients. Specifically, in the low- (less than $7 \mathrm{mmol} / \mathrm{L}$ ) and high- (more than $9 \mathrm{mmol} / \mathrm{L}$ ) glucose groups, lactate was strongly associated with in-hospital mortality and could provide a good performance in predicting inhospital mortality. In the medium-glucose group (between $7 \mathrm{mmol} / \mathrm{L}$ and $9 \mathrm{mmol} / \mathrm{L}$ ), however, lactate was unassociated with in-hospital mortality and its predictive ability reduced significantly. Moreover, we also demonstrated that the APACHE II score and SOFA score were correlated with higher in-hospital mortality in all three glucose groups. Therefore, in patients with glucose levels between 7 and $9 \mathrm{mmol} / \mathrm{L}$ where the lactate level was inadequate to predict in-hospital mortality, the SOFA score and APACHE II score could be utilized as alternative indexes in order to achieve a more accurate prediction. 
TABLE 3: Univariable and multivariable analysis of in-hospital mortality based on glucose levels.

(a) Glucose $<7 \mathrm{mmol} / \mathrm{L}$

\begin{tabular}{|c|c|c|c|c|}
\hline \multirow{2}{*}{ Variable } & \multicolumn{2}{|c|}{ Univariable regression } & \multicolumn{2}{|c|}{ Multivariable regression } \\
\hline & OR (95\%-CI) & $p$ value & OR $(95 \%-C I)$ & $p$ value \\
\hline Age & $0.507(0.221-1.163)$ & 0.109 & & \\
\hline Gender & $0.492(0.214-1.132)$ & 0.095 & & \\
\hline \multicolumn{5}{|l|}{ Reason for ICU admission } \\
\hline Cardiovascular disease & $1.796(0.701-4.601)$ & 0.222 & & \\
\hline Gastrointestinal disease & $0.879(0.364-2.121)$ & 0.773 & & \\
\hline Neurological disease & $0.969(0.300-3.315)$ & 0.959 & & \\
\hline Respiratory disease & $1.447(0.609-3.441)$ & 0.403 & & \\
\hline Surgery & $1.360(0.593-3.118)$ & 0.467 & & \\
\hline Sepsis & $1.040(0.320-3.383)$ & 0.948 & & \\
\hline Trauma & $0.519(0.112-2.406)$ & 0.402 & & \\
\hline Other & $2.917(0.877-9.695)$ & 0.081 & & \\
\hline \multicolumn{5}{|l|}{ Comorbidities } \\
\hline ACS & $0.914(0.187-4.475)$ & 0.911 & & \\
\hline AKI & $1.162(0.423-3.192)$ & 0.771 & & \\
\hline ALI & $0.874(0.234-3.266)$ & 0.841 & & \\
\hline ARDS & $2.619(0.938-7.314)$ & 0.066 & & \\
\hline Cirrhosis & $0.393(0.048-3.199)$ & 0.383 & & \\
\hline Diabetes mellitus & $3.363(0.984-11.498)$ & 0.053 & & \\
\hline DIC & $4.250(0.258-70.045)$ & 0.312 & & \\
\hline Drinking & $0.180(0.041-0.798)$ & 0.024 & $0.157(0.018-1.399)$ & 0.097 \\
\hline Hypertension & $0.634(0.238-1.690)$ & 0.362 & & \\
\hline Malignant disease & $2.308(0.882-6.043)$ & 0.089 & & \\
\hline MOF & $2.550(1.003-6.481)$ & 0.049 & $1.443(0.475-4.386)$ & 0.517 \\
\hline Smoking & $0.365(0.139-0.962)$ & 0.042 & $0.484(0.115-2.041)$ & 0.323 \\
\hline Stroke & $0.613(0.169-2.232)$ & 0.458 & & \\
\hline APACHE II & $1.083(1.027-1.142)$ & 0.003 & $1.043(0.969-1.123)$ & 0.262 \\
\hline Lactate & $1.126(1.042-1.216)$ & 0.003 & $1.093(1.007-1.188)$ & 0.035 \\
\hline qSOFA & $1.356(0.862-2.132)$ & 0.188 & & \\
\hline SOFA & $1.147(1.043-1.262)$ & 0.005 & $1.104(1.006-1.210)$ & 0.036 \\
\hline
\end{tabular}

(b) $7 \mathrm{mmol} / \mathrm{L} \leq$ glucose $\leq 9 \mathrm{mmol} / \mathrm{L}$

\begin{tabular}{lcc}
\hline Variable & $\begin{array}{c}\text { Univariable regression } \\
\text { OR }(95 \%-C I)\end{array}$ & $\begin{array}{c}\text { Multivariable regression } \\
\text { OR }(95 \%-C I)\end{array}$ \\
\hline Age & $0.507(0.221-1.163)$ & 0.109 \\
Gender & $1.067(0.301-3.785)$ & 0.920 \\
Reason for ICU admission & & \\
$\quad$ Cardiovascular disease & $1.387(0.310-6.216)$ & 0.669 \\
Gastrointestinal disease & $0.312(0.087-1.115)$ & 0.073 \\
Neurological disease & $2.400(0.489-11.772)$ & 0.281 \\
Respiratory disease & $0.927(0.216-3.986)$ & 0.919 \\
Surgery & $0.732(0.195-2.749)$ & 0.644 \\
Sepsis & $0.542(0.059-4.956)$ & 0.587 \\
Trauma & $0.841(0.155-4.554)$ & 0.841 \\
Other & - & - \\
\hline
\end{tabular}


TABle 3: Continued.

\begin{tabular}{|c|c|c|c|c|}
\hline \multirow{2}{*}{ Variable } & \multicolumn{2}{|c|}{ Univariable regression } & \multicolumn{2}{|c|}{ Multivariable regression } \\
\hline & OR $(95 \%-C I)$ & $p$ value & OR $(95 \%-C I)$ & $p$ value \\
\hline \multicolumn{5}{|l|}{ Comorbidities } \\
\hline ACS & $1.629(0.356-7.456)$ & 0.530 & & \\
\hline AKI & $0.987(0.179-5.450)$ & 0.988 & & \\
\hline ALI & $4.200(0.735-23.991)$ & 0.107 & & \\
\hline ARDS & $0.841(0.155-4.554)$ & 0.841 & & \\
\hline Cirrhosis & $0.854(0.087-8.383)$ & 0.892 & & \\
\hline Diabetes mellitus & $2.891(0.747-11.192)$ & 0.124 & & \\
\hline DIC & - & - & & \\
\hline Drinking & $0.727(0.136-3.880)$ & 0.709 & & \\
\hline Hypertension & $0.738(0.175-3.124)$ & 0.680 & & \\
\hline Malignant disease & $0.137(0.016-1.152)$ & 0.067 & & \\
\hline MOF & $2.889(0.672-12.415)$ & 0.154 & & \\
\hline Smoking & $1.556(0.395-6.131)$ & 0.528 & & \\
\hline Stroke & $3.909(0.494-30.942)$ & 0.197 & & \\
\hline APACHE II & $1.144(1.027-1.142)$ & 0.005 & $1.126(0.982-1.290)$ & 0.089 \\
\hline Lactate & $1.168(0.928-1.470)$ & 0.186 & & \\
\hline qSOFA & $1.953(0.891-4.279)$ & 0.095 & & \\
\hline SOFA & $1.229(1.033-1.463)$ & 0.020 & $1.041(0.812-1.333)$ & 0.753 \\
\hline
\end{tabular}

(c) Glucose $>9 \mathrm{mmol} / \mathrm{L}$

\begin{tabular}{|c|c|c|c|c|}
\hline \multirow{2}{*}{ Variable } & \multicolumn{2}{|c|}{ Univariable regression } & \multicolumn{2}{|c|}{ Multivariable regression } \\
\hline & OR $(95 \%-C I)$ & $p$ value & OR $(95 \%-C I)$ & $p$ value \\
\hline Age & $1.019(0.984-1.050)$ & 0.288 & & \\
\hline Gender & $3.250(0.965-10.950)$ & 0.057 & & \\
\hline \multicolumn{5}{|l|}{ Reason for ICU admission } \\
\hline Cardiovascular disease & $2.813(0.718-11.021)$ & 0.138 & & \\
\hline Gastrointestinal disease & $0.698(0.214-2.278)$ & 0.552 & & \\
\hline Neurological disease & $1.877(0.509-6.921)$ & 0.344 & & \\
\hline Respiratory disease & $0.977(0.281-3.398)$ & 0.971 & & \\
\hline Surgery & $0.642(0.188-2.188)$ & 0.479 & & \\
\hline Sepsis & $0.727(0.145-3.636)$ & 0.698 & & \\
\hline Trauma & 0 & 0.999 & & \\
\hline Other & $1.047(0.109-10.014)$ & 0.968 & & \\
\hline \multicolumn{5}{|l|}{ Comorbidities } \\
\hline ACS & $3.333(0.823-13.494)$ & 0.091 & & \\
\hline AKI & $0.341(0.041-2.840)$ & 0.320 & & \\
\hline ALI & $0.571(0.066-4.983)$ & 0.613 & & \\
\hline ARDS & $0.492(0.057-4.226)$ & 0.518 & & \\
\hline Cirrhosis & $1.050(0.202-5.460)$ & 0.954 & & \\
\hline Diabetes mellitus & $1.513(0.420-5.447)$ & 0.526 & & \\
\hline DIC & 0 & 0.999 & & \\
\hline Drinking & $1.432(0.439-4.673)$ & 0.552 & & \\
\hline Hypertension & $1.391(0.453-4.268)$ & 0.564 & & \\
\hline Malignant disease & $1.324(0.408-4.295)$ & 0.641 & & \\
\hline MOF & $1.476(0.353-6.166)$ & 0.593 & & \\
\hline Smoking & $1.062(0.332-3.404)$ & 0.919 & & \\
\hline Stroke & $1.476(0.353-6.166)$ & 0.593 & & \\
\hline
\end{tabular}


TABLE 3: Continued.

\begin{tabular}{lccc}
\hline Variable & \multicolumn{2}{c}{ Univariable regression } & \multicolumn{2}{c}{ Multivariable regression } \\
& OR $(95 \%-C I)$ & $p$ value $(95 \%-C I)$ & $1.056(0.962-1.158)$ \\
APACHE II & $1.087(1.017-1.161)$ & $\mathbf{0 . 0 1 4}$ & $1.118(0.997-1.255)$ \\
Lactate & $1.154(1.035-1.285)$ & $\mathbf{0 . 0 1 0}$ & 0.252 \\
qSOFA & $1.783(0.940-3.383)$ & 0.077 & 0.057 \\
SOFA & $1.157(1.026-1.305)$ & $\mathbf{0 . 0 1 7}$ & $1.039(0.876-1.233)$ \\
\hline
\end{tabular}

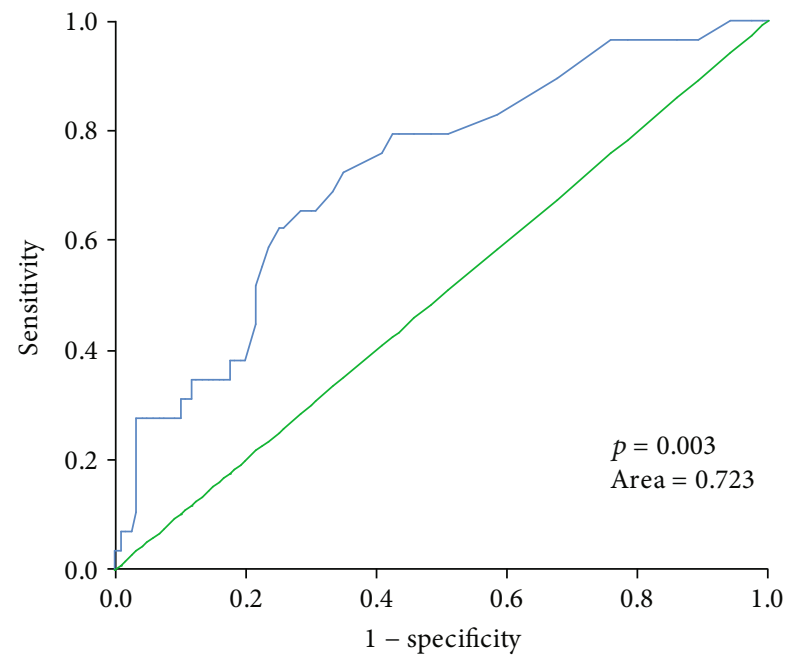

(a)

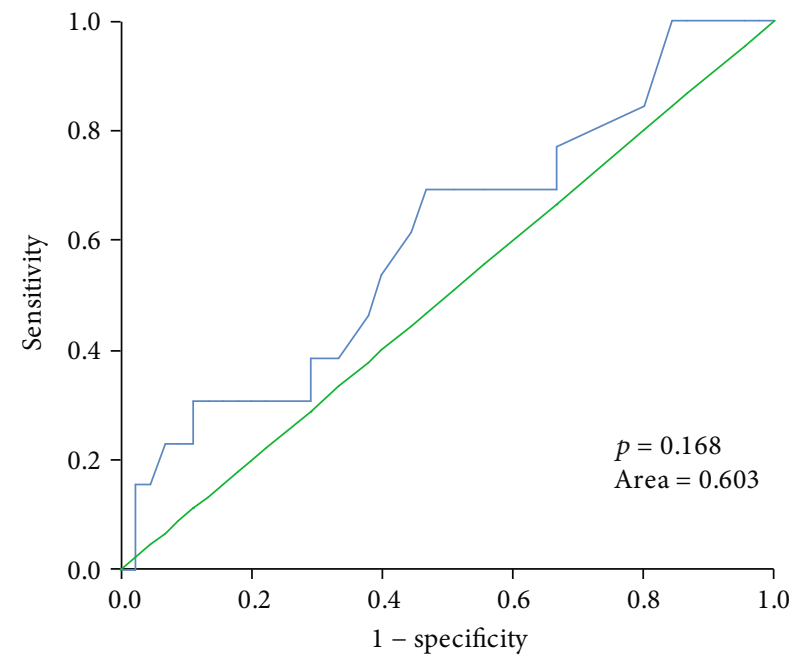

(b)

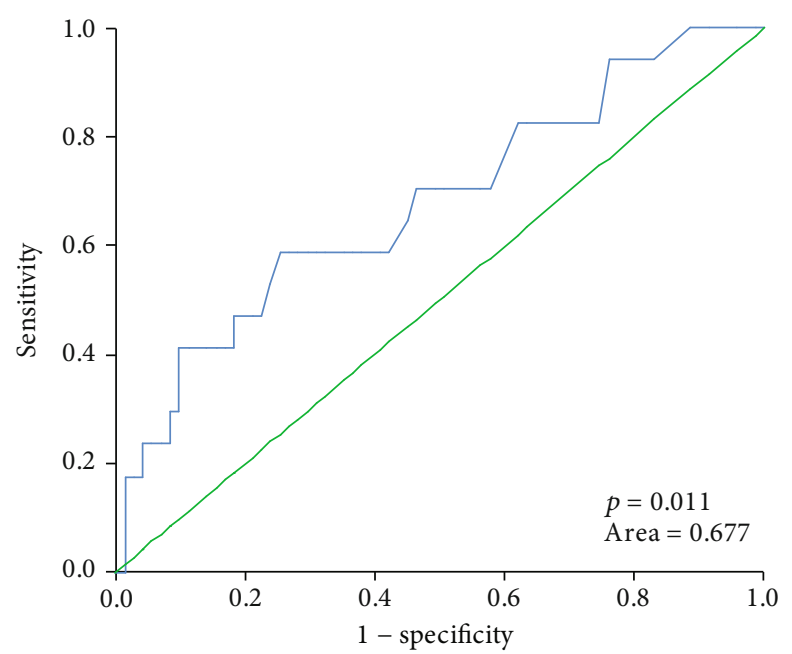

(c)

FIGURE 3: ROC of lactate in the three glucose groups: (a) the ROC of lactate in the low-glucose group (glucose $<7 \mathrm{mmol} / \mathrm{L}$ ) with an AUROC of 0.723 ; (b) the ROC of lactate in the medium-glucose group $(7 \mathrm{mmol} / \mathrm{L} \leq$ glucose $\leq 9 \mathrm{mmol} / \mathrm{L})$ with an AUROC of 0.603 ; (c) the ROC of lactate in the high-glucose group (glucose $>9 \mathrm{mmol} / \mathrm{L}$ ) with an AUROC of 0.677 .

Glucose and lactate intervene in a very complex way in a series of glycometabolic pathways and play important roles in glycometabolic related diseases. Plenty of observational studies have demonstrated that the glucose level during ICU admission was related to mortality by a U-shaped curve $[11,12]$. Both hyperglycemia and hypoglycemia had an increased risk of death and were associated with an increase in the ICU length of stay [13-16]. The "safe range" of glucose has been defined between 7.0 and $9.0 \mathrm{mmol} / \mathrm{L}$ which is within the optimal blood glucose concentration (BGC) target range (NICE-SUGAR protocol) [12,13]. The mean glucose values between 7.0 and $9.0 \mathrm{mmol} / \mathrm{L}$ during ICU stay were associated with the lowest OR for mortality at the ICU, while the mean values below 7.0 and higher than $9.0 \mathrm{mmol} / \mathrm{L}$ conferred significantly higher ORs [12].

Lactate has also been widely studied as a biomarker of the outcomes of a series of disease related to tissue hypoxia, sepsis, and organ failure $[1,3-5,7-10,17,22]$. Bakker et al. 


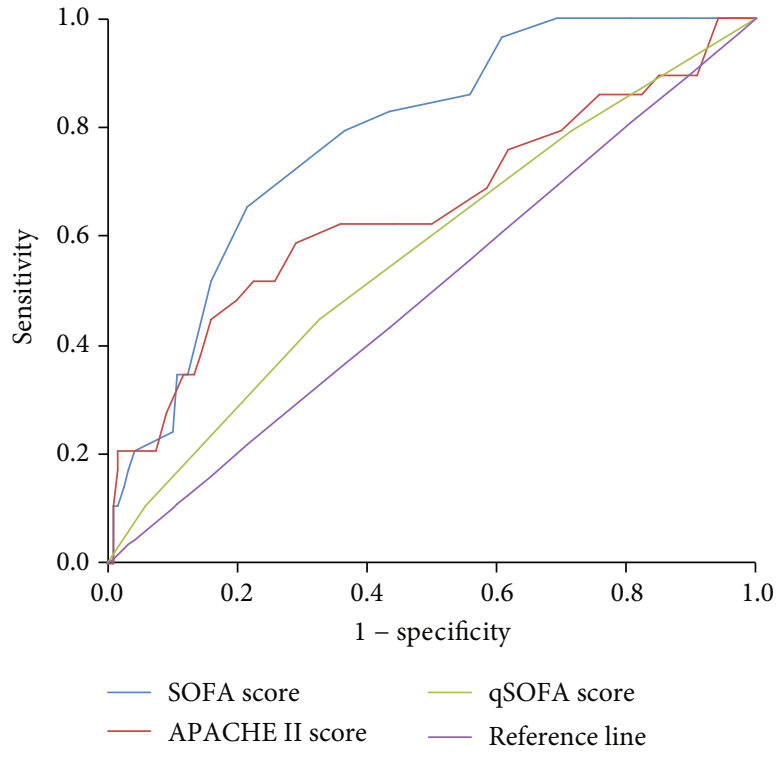

(a)

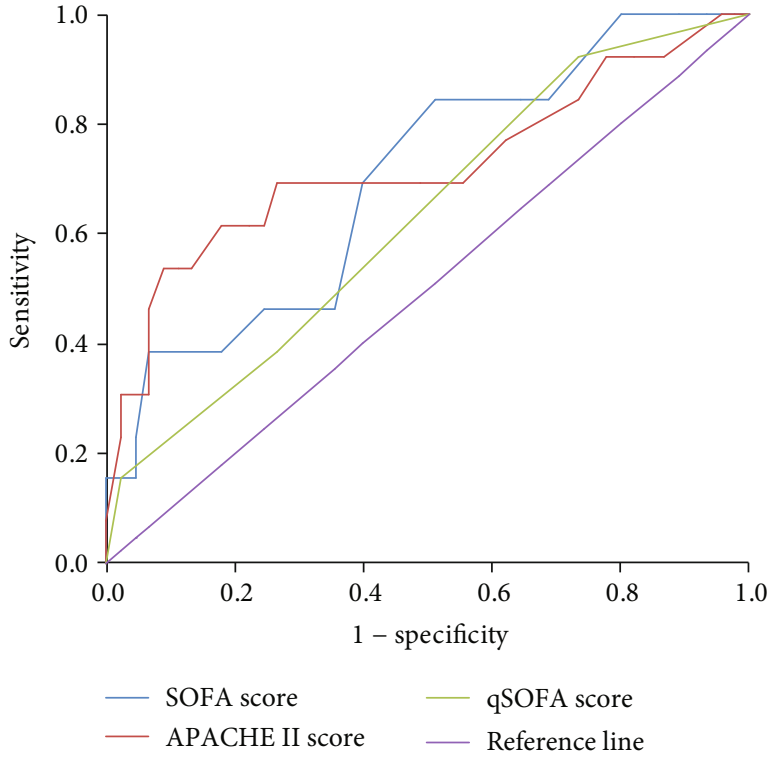

(b)

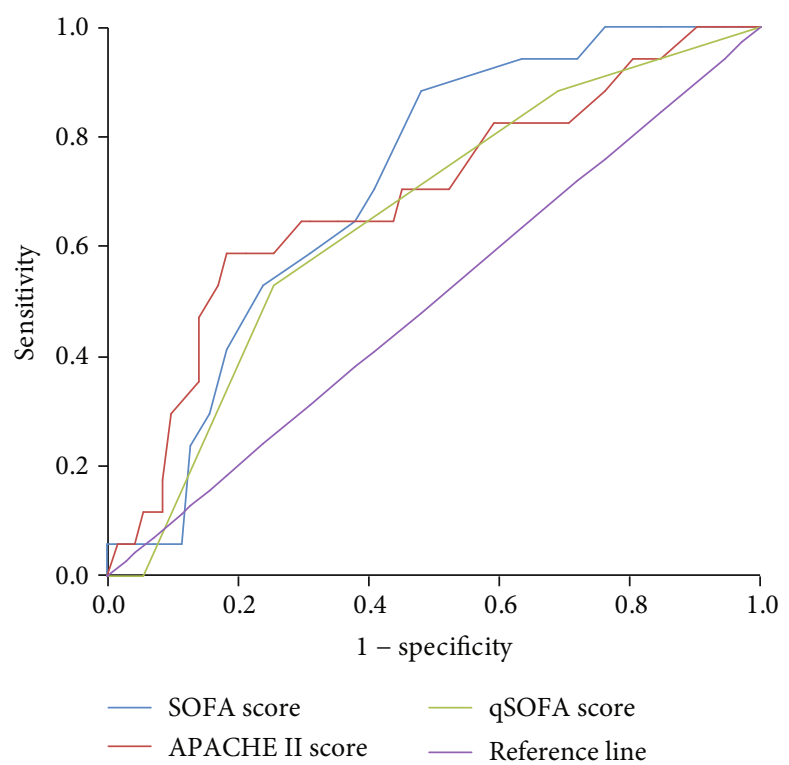

(c)

FIgURE 4: ROC of the APACHE II score, SOFA score, and qSOFA in the three glucose groups: (a) in the low-glucose group (glucose $<7 \mathrm{mmol} / \mathrm{L}$ ), the AUROC of the APACHE II score, SOFA score, and qSOFA score were 0.650, 0.779, and 0.574, respectively; (b) in the medium-glucose group $(7 \mathrm{mmol} / \mathrm{L} \leq$ glucose $\leq 9 \mathrm{mmol} / \mathrm{L})$, the AUROC of the APACHE II score, SOFA score, and qSOFA score were $0.723,0.700$, and 0.629 , respectively; (c) in the high-glucose group (glucose $>9 \mathrm{mmol} / \mathrm{L}$ ), the AUROC of the APACHE II score, SOFA score, and qSOFA score were $0.690,0.712$, and 0.652 , respectively.

reported that patients with lower organ failure scores had a lower initial blood lactate level and a shorter duration of lactic acidosis, while patients who died during the first 24 hours had a higher initial blood lactate level and a longer duration of lactic acidosis [9]. Amorini et al. utilized serum lactate as a monitoring of "virtual hypoxia" in multiple sclerosis patients and a secondary outcome for treatment trials aimed at improving mitochondrial function in patients with multiple sclerosis [10]. Krinsley et al. demonstrated that the hypoglycemia could be the result of impaired renal and liver function which affects outcomes [4]. Filho et al. reported the utilization of cerebrospinal fluid (CSF) lactate as a potential biomarker to distinguish between children with bacterial and aseptic meningitis [5]. Mokline et al. reported that the plasma lactate could be used as a powerful predictor biomarker of sepsis and mortality in burn patients. An initial lactate value of $4 \mathrm{mmol} / \mathrm{L}$ provided the best sensitivity (88\%) and specificity (79\%), and the cutoff value for mortality prediction was $4.46 \mathrm{mmol} / \mathrm{L}$ with a good sensitivity (86\%) and specificity (92\%) [3]. Mikkelsen et al. demonstrated that the initial serum lactate was associated with mortality in patients admitted to the ED with severe sepsis [8]. Shapiro 
et al. demonstrated that lactate level greater than or equal to $4.0 \mathrm{mmol} / \mathrm{L}$ had good sensitivity and specificity to predict the mortality within 3 days [7].

Many effects have been done in investigating the relations of lactate and glucose with the outcomes of ICU patients; however, the combined effect of glucose and lactate has rarely been well studied. A recent study conducted by Jorge et al. investigated the relation between the combined glucose/lactate and mortality as well as organ failure. They found that the combination of the highest lactate quintile with the lowest glucose quintile was associated with the highest rates of renal dysfunction, liver dysfunction, and mortality [11]. In addition to glucose, interactions between lactate and other parameters have also been studied. Ospina-Tascón et al. demonstrated that the combined $\mathrm{Cv}-\mathrm{aCO}_{2} / \mathrm{Da}-\mathrm{vO}_{2}$ ratio with lactate levels could better identify patients at a high risk of adverse outcomes during early stages of resuscitation of septic shock [23]. Ho and Lan reported that combining the qSOFA score with plasma lactate had a predictive ability comparable to the standard SOFA score [24].

Our study substantially extends these previous investigations and presented a comprehensive view of how the overall glycometabolic metabolism affects the outcomes of ICU patients. We demonstrated that prediction of in-hospital mortality of ICU patient is glycometabolism related rather than only lactate related. Neither glucose nor lactate could be used as a predictor of the in-hospital mortality independently. In the low- and high-glucose groups, lactate was associated significantly with in-hospital mortality, whereas in the medium-glucose group, lactate was inadequate to provide a good prediction of in-hospital mortality and other wellaccepted indexes such as the APACHE II score and SOFA score should be utilized to obtain a more reasonable and accurate assessment of in-hospital mortality.

Nevertheless, some limitations could still be seen in our study. Due to the small number of clinical samples, selection bias might be inevitable and influence the statistical results. It is interesting to note that in the low- (less than $7 \mathrm{mmol} / \mathrm{L}$ ) glucose group, diabetes mellitus has a near significant $p$ value (0.053), indicating that at low glucose levels, diabetes mellitus may be also associated with the in-hospital mortality. This finding is consistent with previous demonstration that the low glucose is associated with the higher risk of death [2527]. Therefore, we envisioned that with larger populations, a more reasonable and comprehensive demonstration could be generated. Moreover, as it is retrospective and one center study, prospective and multicenter researches would be required in the future to further validate our conclusions.

\section{Conclusion}

In conclusion, our results showed that the predictive ability of lactate to assess in-hospital mortality also depended on glucose levels. In low and high glucose levels, lactate level would provide a good prediction of in-hospital mortality while in the medium glucose level, the APACHE II score or SOFA score could provide a more accurate and more comprehensive prediction of in-hospital mortality.

\section{Abbreviations}

$\begin{array}{ll}\text { ACS: } & \text { Acute coronary syndrome } \\ \text { AKI: } & \text { Acute kidney injury } \\ \text { ALI: } & \text { Acute lung injury } \\ \text { APACHE II: } & \text { Acute Physiology and Chronic Health } \\ & \text { Evaluation II } \\ \text { ARDS: } & \text { Acute respiratory distress syndrome } \\ \text { AUROC: } & \text { Area under receiver operating charac- } \\ & \text { teristic curve } \\ \text { CAD: } & \text { Coronary artery disease } \\ \text { Cv-aCO } / \mathrm{Da}_{2}: \mathrm{O}_{2}: & \text { Ratio of venous-to-arterial carbon diox- } \\ & \text { ide content difference to arterial-to- } \\ & \text { venous oxygen content difference } \\ \text { DE: } & \text { Emergency department } \\ \text { DIC: } & \text { Disseminated intravascular coagulation } \\ \text { ICU: } & \text { Intensive care unit } \\ \text { MOF: } & \text { Multiple organ failure } \\ \text { qSOFA: } & \text { Quick Sequential Organ Failure } \\ \text { ROC: } & \text { Assessment } \\ \text { SOFA: } & \text { Receiver operating characteristic curve } \\ & \text { Sequential Organ Failure Assessment. }\end{array}$

\section{Data Availability}

The subsets of the data sets used and/or analyzed during the current study are available from the corresponding author on reasonable request.

\section{Ethical Approval}

The anonymized data analysis in this study was performed in accordance with the guidelines outlined in Chinese legislation, and the study was approved by the medical ethics committee of our institution (First Affiliated Hospital of Xi'an Jiaotong University). Because this study was a retrospective study of routinely collected data, informed consent was not required by our ethics committee.

\section{Conflicts of Interest}

The authors declare that they have no competing interests.

\section{Authors' Contributions}

Chen X participated in the research design, data collection, statistical analysis, and paper writing. Bi J participated in the data collection and statistical analysis. Zhang J, Ren Y, and Wei S participated in the data collection. Du Z participated in the statistical analysis. $\mathrm{Wu} \mathrm{Z}$ and $\mathrm{Lv} \mathrm{Y}$ assisted with the design of the study and analyzed the data. Wu R designed and supervised the study and revised the manuscript. Xue Chen and Jianbin Bi contributed equally to this work.

\section{Acknowledgments}

The work was supported by grants from the National Natural Science Foundation of China (No. 81770491) and the Ministry of Education Innovation Team Development Program of China (No. IRT16R57) and a research fund for the Young 
Talent Recruiting Plans of Xi'an Jiaotong University (RW). The authors kindly thank the Department of Clinical Information Management of The First Affiliated Hospital of Xi' an Jiaotong University for providing the ICU patient's clinical information.

\section{Supplementary Materials}

Table S1: patient number of 6 subgroups. (Supplementary Materials)

\section{References}

[1] S. Robertson, E. Dickson, and G. Richards, "The nonlactate gap: a novel predictor of organ failure and mortality following major trauma," Critical Care, vol. 11, article P346, Supplement 2, 2007.

[2] N. T. Huy, N. T. H. Thao, D. T. N. Diep, M. Kikuchi, J. Zamora, and K. Hirayama, "Cerebrospinal fluid lactate concentration to distinguish bacterial from aseptic meningitis: a systemic review and meta-analysis," Critical Care, vol. 14, no. 6, article R240, 2010.

[3] A. Mokline, A. Abdenneji, I. Rahmani et al., "Lactate: prognostic biomarker in severely burned patients," Annals of Burns \& Fire Disasters, vol. 30, no. 1, pp. 35-38, 2017.

[4] J. Krinsley, M. S. Schultz, P. Spronk et al., "Mild hypoglycemia is independently associated with increased mortality in the critically ill," Critical Care, vol. 15, article P397, Supplement 1, 2011.

[5] F. M. Filho, S. M. Horita, A. E. Gilio, and L. E. Nigrovic, "Cerebrospinal fluid lactate level as a diagnostic biomarker for bacterial meningitis in children," International Journal of Emergency Medicine, vol. 7, no. 1, p. 14, 2014.

[6] T. C. Jansen, J. van Bommel, F. J. Schoonderbeek et al., "Early lactate-guided therapy in intensive care unit patients: a multicenter, open-label, randomized controlled trial," American Journal of Respiratory and Critical Care Medicine, vol. 182, no. 6, pp. 752-761, 2010.

[7] N. I. Shapiro, M. D. Howell, D. Talmor et al., "Serum lactate as a predictor of mortality in emergency department patients with infection," Annals of Emergency Medicine, vol. 45, no. 5, pp. 524-528, 2005.

[8] M. E. Mikkelsen, A. N. Miltiades, D. F. Gaieski et al., "Serum lactate is associated with mortality in severe sepsis independent of organ failure and shock," Critical Care Medicine, vol. 37, no. 5, pp. 1670-1677, 2009.

[9] J. Bakker, P. Gris, M. Coffernils, R. J. Kahn, and J. L. Vincent, "Serial blood lactate levels can predict the development of multiple organ failure following septic shock," American Journal of Surgery, vol. 171, no. 2, pp. 221-226, 1996.

[10] A. M. Amorini, V. Nociti, A. Petzold et al., "Serum lactate as a novel potential biomarker in multiple sclerosis," Biochimica et Biophysica Acta (BBA) - Molecular Basis of Disease, vol. 1842, no. 7, pp. 1137-1143, 2014.

[11] P. F. Jorge, N. Wieringa, E. de Felice, I. C. C. van der Horst, A. O. Lansink, and M. W. Nijsten, "The association of early combined lactate and glucose levels with subsequent renal and liver dysfunction and hospital mortality in critically ill patients," Critical Care, vol. 21, no. 1, p. 218, 2017.

[12] S. E. Siegelaar, J. Hermanides, O. V. Straaten et al., Mean Glucose during ICU Admission Is Related to Mortality by a UShaped Curve: Implications for Clinical Care, vol. 2010, Meeting of the American-Diabetes-Association, 2010.
[13] F. Bilotta and G. Rosa, "Glycemia management in critical care patients," World Journal of Diabetes, vol. 3, no. 7, pp. 130-134, 2012.

[14] M. Egi, R. Bellomo, E. Stachowski et al., "Hypoglycemia and outcome in critically ill patients," Mayo Clinic Proceedings, vol. 85, no. 3, pp. 217-224, 2010.

[15] J. Krinsley, M. J. Schultz, P. E. Spronk et al., "Mild hypoglycemia is strongly associated with increased intensive care unit length of stay," Annals of Intensive Care, vol. 1, no. 1, p. 49, 2011.

[16] A. T. Schlussel, D. B. Holt, E. A. Crawley, M. B. Lustik, C. E. Wade, and C. F. T. Uyehara, "Effects of hyperglycemia and continuous intravenous insulin on outcomes of surgical patients," Journal of Surgical Research, vol. 176, no. 1, pp. 202-209, 2012.

[17] S. B. Lee, Y. S. Hong, J. Y. Kim et al., "Lactate clearance as a prognostic factor of organ failure in sepsis," Journal of The Korean Society of Emergency Medicine, vol. 20, no. 6, pp. 642-648, 2009.

[18] J. L. Vincent, R. Moreno, J. Takala et al., "The SOFA (Sepsisrelated Organ Failure Assessment) score to describe organ dysfunction/failure: on behalf of the Working Group on SepsisRelated Problems of the European Society of Intensive Care Medicine," Intensive Care Medicine, vol. 22, no. 7, pp. 707$710,1996$.

[19] H. Gomez and J. A. Kellum, "Lactate in sepsis," JAMA, vol. 313, no. 2, pp. 194-195, 2015.

[20] M. Singer, C. S. Deutschman, C. W. Seymour et al., "The third international consensus definitions for sepsis and septic shock (sepsis-3)," JAMA, vol. 315, no. 8, pp. 801-810, 2016.

[21] J. A. Kellum, N. Lameire, and for the KDIGO AKI Guideline Work Group, "Diagnosis, evaluation, and management of acute kidney injury: a KDIGO summary (part 1)," Critical Care, vol. 17, no. 1, p. 204, 2013.

[22] C. Chiarla, I. Giovannini, and G. Miggiano, "Plasma amino acids of the transsulfuration pathway and plasma lactate in septic patients," La Clinica Terapeutica, vol. 168, no. 2, pp. e72-e76, 2017.

[23] G. A. Ospina-Tascón, M. Umaña, W. Bermúdez et al., "Combination of arterial lactate levels and venous-arterial $\mathrm{CO}_{2}$ to arterial-venous $\mathrm{O}_{2}$ content difference ratio as markers of resuscitation in patients with septic shock," Intensive Care Medicine, vol. 41, no. 5, pp. 796-805, 2015.

[24] K. M. Ho and N. S. H. Lan, "Combining quick Sequential Organ Failure Assessment with plasma lactate concentration is comparable to standard Sequential Organ Failure Assessment score in predicting mortality of patients with and without suspected infection," Journal of Critical Care, vol. 38, pp. 1-5, 2017.

[25] The Action to Control Cardiovascular Risk in Diabetes Study Group, "Effects of intensive glucose lowering in type 2 diabetes," The New England Journal of Medicine, vol. 358, no. 24, pp. 2545-2559, 2008.

[26] M. Wei, L. W. Gibbons, T. L. Mitchell, J. B. Kampert, M. P. Stern, and S. N. Blair, "Low fasting plasma glucose level as a predictor of cardiovascular disease and all-cause mortality," Circulation, vol. 101, no. 17, pp. 2047-2052, 2000.

[27] A. Akirov, A. Grossman, T. Shochat, and I. Shimon, "Mortality among hospitalized patients with hypoglycemia: insulin related and noninsulin related," The Journal of Clinical Endocrinology \& Metabolism, vol. 102, no. 2, pp. 416-424, 2017. 


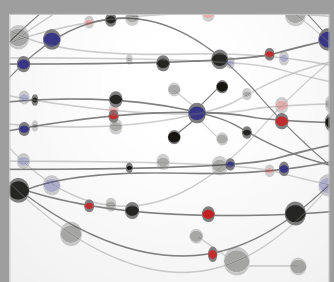

The Scientific World Journal
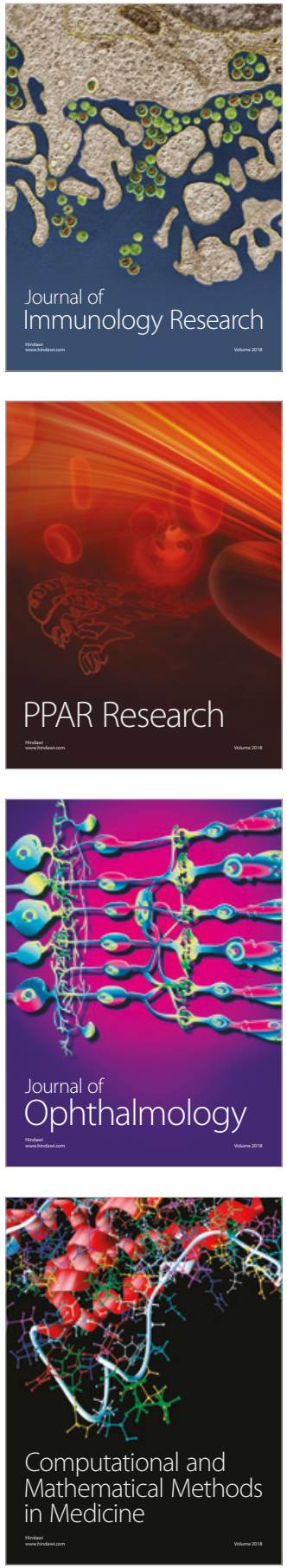

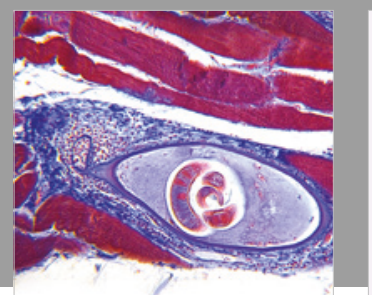

Gastroenterology Research and Practice

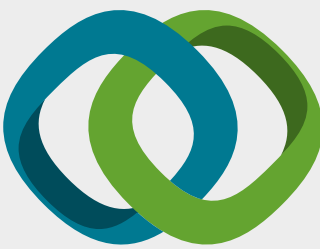

\section{Hindawi}

Submit your manuscripts at

www.hindawi.com
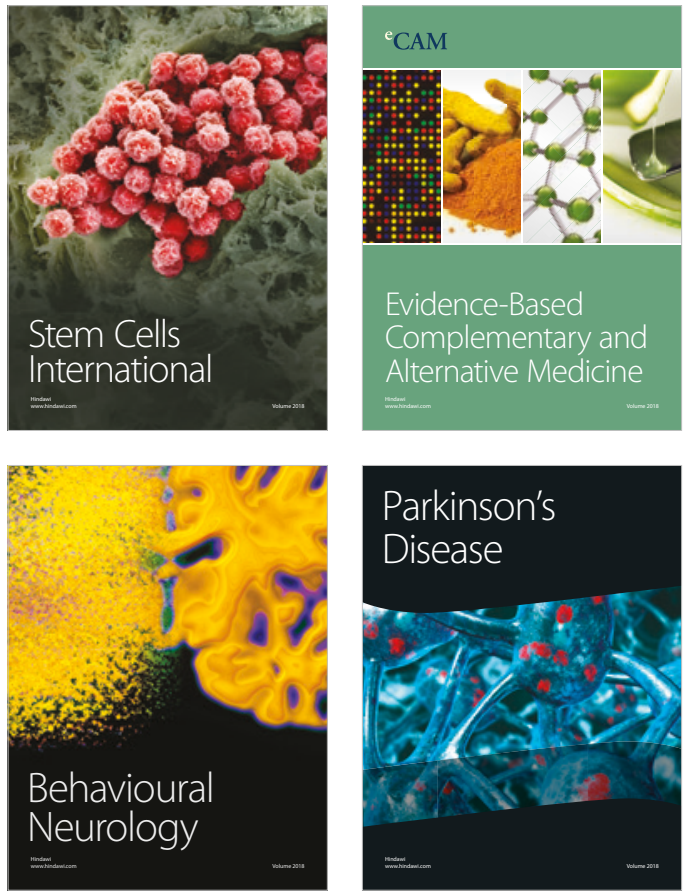

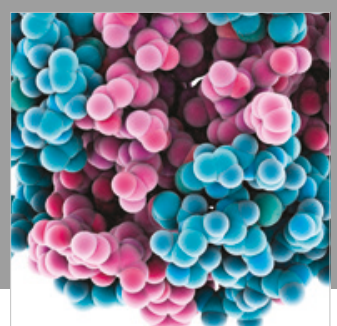

ournal of

Diabetes Research

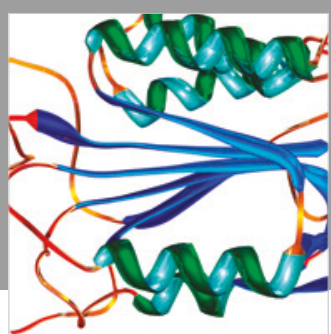

Disease Markers
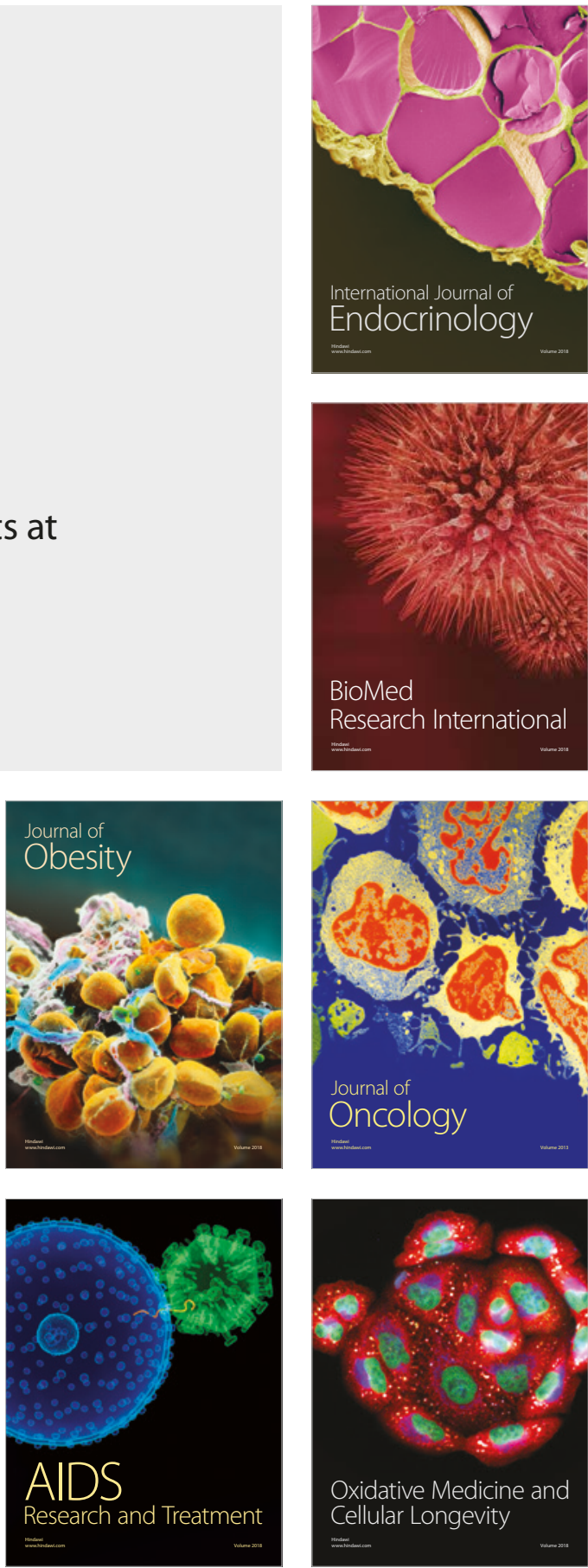